SILVA, AC; AROUCHA, EMM; CHAVES, SWP; MEDEIROS, JF; PAIVA, CA; ARAÚJO, NO. 2016. Efeito de diferentes doses, formas de aplicação e fontes de P na conservação de melancia sem sementes. Horticultura Brasileira 34: 529-536. DOI - http://dx.doi.org/10.1590/S0102-053620160412

\title{
Efeito de diferentes doses, formas de aplicação e fontes de $P$ na conservação de melancia sem sementes
}

\author{
Ana C Silva; Edna MM Aroucha; Sérgio WP Chaves; José F Medeiros; Cristiane A Paiva; Nícolas O \\ Araújo \\ Universidade Federal Rural do Semiárido (UFERSA), Mossoró-RN, Brasil; ana_claudia33@hotmail.com; aroucha@ufersa.edu.br; \\ swchaves@ufersa.edu.br; jfmedeir@ufersa.edu.br; cristiane_uzl@hotmail.com; nicolas_araujo1892@hotmail.com
}

\section{RESUMO}

O trabalho teve como objetivo avaliar o efeito de doses, fontes e formas de aplicação de P na conservação de melancia sem semente. Para isto, foi realizado o plantio da melancia cv. 'Style'. Os tratamentos consistiram de duas doses de $\mathrm{P}\left(80\right.$ e $\left.289 \mathrm{~kg} / \mathrm{ha} \mathrm{P}_{2} \mathrm{O}_{5}\right)$ em duas formas de aplicação (fundação e cobertura), utilizando o superfosfato triplo, na fundação, e o fosfato monoamônico (MAP) em cobertura. Ainda, foi acrescentado um tratamento adicional com a maior dose $\left(289 \mathrm{~kg} / \mathrm{ha}_{2} \mathrm{O}_{5}\right)$ parcelada com MAP em fundação e cobertura, totalizando cinco tratamentos. No laboratório, foi instalado um experimento inteiramente casualizado em esquema fatorial $5 \times 5$, sendo as cinco adubações fosfatadas e cinco períodos de armazenamento dos frutos $(0,14,21,28$ e 35 dias após a colheita), com quatro repetições. Uma amostra de oito frutos foi avaliada no tempo zero e os demais armazenados em temperatura de $9 \pm 2^{\circ} \mathrm{C}$ e $85 \pm 2 \%$ UR, onde permaneceram por $7,14,21$ e 28 dias e mais sete dias à temperatura de $23 \pm 2^{\circ} \mathrm{C}$ e $60 \pm 2 \%$ UR. Foram avaliados nos frutos a massa, comprimento, diâmetro, espessura de polpa, formato, perda de massa (PM), firmeza da polpa (FP), sólidos solúveis (SS), acidez titulável (AT), açúcares solúveis (AS), relação SS/AT e licopeno. A adubação (doses, forma de aplicação e fontes de P) não afetou a massa, comprimento, diâmetro, espessura de polpa, formato do fruto, perda de massa, firmeza de polpa e teor de licopeno dos frutos. Todavia, houve interação entre os fatores adubação e armazenamento, para AS e relação SS/AT. Houve acréscimos na PM e decréscimos nos teores de SS, AS, AT e licopeno dos frutos durante o armazenamento. Porém, a adubação fosfatada, na menor dose $(80 \mathrm{~kg} / \mathrm{ha})$ aplicada via fundação, resultou em melhor resultado na conservação pós-colheita da melancia sem semente, mantendo-se maior teor de AS e relação SS/AT da polpa até 35 dias de armazenamento.

Palavras-chave: Citrullus lanatus, adubação, pós-colheita.

\section{ABSTRACT}

Effect of different doses, application forms and sources of $P$ in the conservation of seedless watermelon

The effect of doses, sources and application forms of $\mathrm{P}$ was evaluated for the conservation of seedless watermelon. With this purpose we planted the watermelon cv. Style. Treatments consisted of two doses of $\mathrm{P}\left(80\right.$ and $\left.289 \mathrm{~kg} / \mathrm{ha} \mathrm{P}_{2} \mathrm{O}_{5}\right)$ in two application forms (foundation and cover), using triple superphosphate on the foundation, and monoammonium phosphate (MAP) on coverage. Moreover, an additional treatment was evaluated, using the highest dose $(289 \mathrm{~kg} /$ ha $\mathrm{P}_{2} \mathrm{O}_{5}$ ) splitted with MAP in the foundation and cover, totalling five treatments. In the lab, a complete randomized factorial research was installed, in a factorial $5 \times 5$, with five phosphate fertilizations and five fruit storage periods $(0,14,21,28$ and 35 days after harvest), with four replications. A sample of eight fruits was evaluated at time zero and the other fruits were stored at a temperature of $9 \pm 2{ }^{\circ} \mathrm{C}$ and $85 \pm 2 \% \mathrm{RH}$, remaining for $7,14,21$ and 28 days and, additional seven days at a temperature of $23 \pm 2{ }^{\circ} \mathrm{C}$ and $60 \pm 2 \% \mathrm{RH}$. We evaluated the fruit mass, length, diameter, pulp thickness, shape, weight loss (PM), pulp firmness (FP), soluble solids (SS), titratable acidity (AT), soluble sugars (AS), relationship SS/AT and lycopene. Fertilization (doses, application form and P sources) did not affect weight, length, diameter, pulp thickness, fruit shape, mass loss, firmness and fruit lycopene content. However, there was interaction between the factors fertilization and storage for AS and SS/AT ratio. There were increases in PM and decreases in the contents of SS, AS, AT and lycopene on fruits during storage. However, phosphorus fertilization on the lowest dose $(80 \mathrm{~kg} / \mathrm{ha})$, applied via foundation, resulted in better results in seedless watermelon post-harvest conservation, maintaining higher levels of AS and SS/AT on pulp up to 35 days of storage.

Keywords: Citrullus lanatus, fertilization, postharvest.

(Recebido para publicação em 24 de março de 2015; aceito em 18 de fevereiro de 2016) (Received on March, 24, 2015; accepted on February 18, 2016)

\begin{abstract}
A melancia (Citrullus lanatus) é a quarta hortaliça de maior importância no cenário nacional. No ano de 2013 foram produzidos 2.163.501 t, em uma área colhida de 92.021 ha, com rendimento médio de $23,51 \mathrm{t} / \mathrm{ha}$.
\end{abstract}

O Nordeste brasileiro tem expressiva participação nesse agronegócio. Sendo o Rio Grande do Norte o segundo maior produtor (121.047 t) de melancia em área colhida (4.697 ha) e, a Bahia o primeiro, com $212.248 \mathrm{t}$ em uma área de 10.828 t (IBGE, 2015).

O cultivo de melancia sem semente cresce em todo o país, devido à grande demanda pelo mercado internacional, o que está associado às suas boas características como: peso, firmeza de polpa, 
sólidos solúveis e licopeno, que garantem qualidade sensorial e bom manuseio pós-colheita (Leskovar et al., 2004).

As plantas de melancia triplóides (sem semente) são geralmente maiores que as diplóides (com semente) e, como apresentam frutos menores, concentram mais sólidos solúveis (Maynard et al., 2002). Todavia, tanto o desenvolvimento das plantas quanto a produtividade e qualidade dos frutos sofre influência da nutrição mineral do solo (Barros et al., 2012). E, sendo o fósforo um nutriente essencial, esse deve estar em quantidades adequadas, para a planta completar seu ciclo vital (Priya \& Sahi, 2009).

Por outro lado, $30 \%$ dos solos agrícolas mundiais são deficientes em P e requerem aplicação deste para garantir a produtividade e qualidade dos produtos (MacDonald et al., 2011). Todavia, a baixa disponibilidade do P nos solos tropicais associada à sua pouca mobilidade no solo, alta afinidade por óxidos de ferro e alumínio (Novais et al., 2007), aumenta a necessidade de sua incorporação em maior quantidade nos solos.

Os estudos sobre o efeito da adubação fosfatada na qualidade e produtividade de frutos são controversos e sua resposta varia com a espécie (Martuscelli et al., 2015). Informações sobre o impacto da aplicação de fósforo na qualidade e potencial de conservação de melancia sem semente ainda inexistem na literatura.

Há evidências que a adubação fosfatada influencia a produtividade comercial e total bem como o teor de sólidos solúveis e relação SS/AT de melão; tendo o ácido fosfórico propiciado frutos com sólidos solúveis (12,53\%) superior aqueles com superfosfato simples aplicado de modo convencional $(10,92 \%)$ e MAP (10,60\%) (Brito et al., 2000).

A melancia, na região do Agropolo Mossoró-Açu ( $\mathrm{RN})$, é colhida com teor de sólidos solúveis mínimo de $9 \%$ e, para a sua comercialização em mercados distantes, as empresas agrícolas utilizam temperatura de refrigeração $\left(9^{\circ} \mathrm{C}\right.$ e $80 \%$ UR), que é eficiente em retardar o metabolismo que conduz à senescência (Fennema, 2000). As melancias armazenadas em temperatura de $10^{\circ} \mathrm{C}$ e $96 \%$ UR, mantém boa qualidade por até 25 dias (Carlos et al., 2002). Porém, em temperatura ambiente há rápida perda de firmeza de polpa e a vida útil é menor (12 dias) (Araújo Neto et al., 2000).

Tendo em vista a importância da adubação fosfatada para a cultura, e a importância econômica desse fruto para a região do semiárido, este trabalho teve por objetivo avaliar o impacto do cultivo da melancia sem semente ('Style') em diferentes doses, fontes e forma de aplicação da adubação fosfatada na qualidade e vida útil pós-colheita dos frutos sob refrigeração.

\section{MATERIAL E MÉTODOS}

O experimento foi conduzido de setembro a novembro de 2013, localizado no município de Upanema-RN $\left(5^{\circ} 35^{\prime} 04^{\prime \prime}\right.$ S e $37^{\circ} 12^{\prime} 08^{\prime \prime} \mathrm{O}$, altitude 123 $\mathrm{m})$. O clima da região, segundo a classificação de Köppen, é do tipo BSwh' (quente e seco); com precipitação pluviométrica bastante irregular, média anual de 469,8 mm (CPRM, 2015).

O solo da área experimental foi classificado como Cambissolo (Embrapa, 1999), com as seguintes características químicas: $\mathrm{pH}\left(\mathrm{H}_{2} \mathrm{O}\right)=7,4$; Matéria orgânica $=23,86 \mathrm{~g} / \mathrm{kg} ; \mathrm{P}$ Mehlich $=4$ $\mathrm{mg} / \mathrm{dm}^{3} ; \mathrm{K}=5,60 ; \mathrm{Na}=0,70 ; \mathrm{Ca}=48,4$; $\mathrm{Mg}=21,1 ; \mathrm{Al}$ trocável $=0,0 ; \mathrm{H}+\mathrm{Al}=$ $14,9\left(\mathrm{mmolc} / \mathrm{dm}^{3}\right)$ e $\mathrm{V}=84 \%$.

$\mathrm{O}$ experimento foi dividido em duas etapas: campo e laboratório. No campo, foi conduzido em blocos casualizados com quatro repetições. Os tratamentos consistiram na combinação de duas doses de fósforo ( 80 e $289 \mathrm{~kg} / \mathrm{ha} \mathrm{P}_{2} \mathrm{O}_{5}$ ) e duas formas de aplicação (fundação e parcelada, em fundação e cobertura). Utilizou-se como fonte de fósforo o superfosfato triplo $\left(41 \%\right.$ de $\left.\mathrm{P}_{2} \mathrm{O}_{5}\right)$, na fundação, e o fosfato monoamônico (MAP) $\left(48 \%\right.$ de $\left.\mathrm{P}_{2} \mathrm{O}_{5}\right)$, na cobertura além de um tratamento adicional com a maior dose de fósforo $\left(289 \mathrm{~kg} / \mathrm{ha} \mathrm{P}_{2} \mathrm{O}_{5}\right)$, utilizando MAP de forma parcelada. A dose de fósforo aplicada na cobertura foi de $50 \mathrm{~kg} / \mathrm{ha} \mathrm{P}_{2} \mathrm{O}_{5}$, via fertirrigação. No laboratório, foi instalado um experimento inteiramente casualizado em esquema fatorial $5 \times 5$, sendo cinco adubações fosfatadas e cinco períodos de armazenamento dos frutos $(0,14$, 21, 28 e 35 dias após a colheita), com quatro repetições.

A adubação de fundação foi realizada manualmente, (a $30 \mathrm{~cm}$ de cada emissor, a $10 \mathrm{~cm}$ de profundidade). Junto às doses de fósforo foram aplicados ainda $100 \mathrm{~kg} / \mathrm{ha}$ de Barimicro $^{\circledR}$ (FTE BR12), contendo $1,8 \%$ de $\mathrm{B} ; 0,8 \%$ de $\mathrm{Cu} ; 2,0 \%$ de $\mathrm{Mn} ; 9,0 \%$ de $\mathrm{Zn} ;$ e $4,0 \%$ de $\mathrm{S}$.

A cultivar de melancia utilizada foi sem sementes, híbrido 'Style' (triploide). Também utilizou-se uma cultivar não comercial (diploide) como polinizadora. A semeadura foi realizada em bandejas de 200 células e aos 14 dias após a semeadura (DAS) as mudas foram transplantadas para o campo. $\mathrm{O}$ espaçamento utilizado para o plantio foi de 1,9x0,6 m, com uma muda por cova, na proporção de duas 'Style' para uma polinizadora. A área das parcelas experimentais consistiu de 16 plantas de 'Style', sendo a área útil com 10 plantas.

O sistema de irrigação foi o localizado por gotejamento, utilizando um gotejador por planta. O sistema de fertirrigação foi composto por dois tanques de derivação ("pulmões"), conectados a redes de distribuição de água independentes, onde um aplicava os adubos nos tratamentos de fundação e o outro nos tratamentos de fundação mais cobertura (Paula et al., 2011).

As melancias foram colhidas aos 63 DAT e em seguida transportadas para o Laboratório de Tecnologia de Alimentos da UFERSA, após limpeza e pesagem. Uma amostra de oito frutos foi avaliada no tempo zero e os demais foram armazenados por períodos de 7, 14, 21 e 28 dias em câmara de refrigeração regulada a $9 \pm 2{ }^{\circ} \mathrm{C}$ e $85 \pm 2 \%$ UR. Ao fim de cada período, eram armazenados por sete dias à temperatura de $23 \pm 2^{\circ} \mathrm{C}$ e $60 \pm 2 \%$ UR, simulando o período de comercialização na gôndola do supermercado.

Nos frutos foram avaliados a massa média $(\mathrm{kg})$, determinada por gravimetria em balança semi-analítica; comprimento $(\mathrm{cm})$, diâmetro de frutos $(\mathrm{cm})$, espessura de polpa $(\mathrm{cm})$, utilizando-se régua milimetrada; formato de frutos $(\mathrm{F})$, expresso pela relação comprimento/diâmetro, perda de massa (PM) expressa pela diferença entre a massa no tempo inicial e a obtida em cada época, expressa em porcentagem (\%); firmeza da polpa (FP). Os frutos foram divididos em duas 
partes, e procedeu-se três leituras, feitas na região equatorial da polpa, com um penetrômetro da marca McCormick, modelo FT 327 analógico (ponteira de $12 \mathrm{~mm}$ de diâmetro), e expresso em Newton (N); sólidos solúveis (SS), determinado com um refratômetro digital modelo PR-100 Palette (Attago Co. Ltda., Japan). Utilizou-se uma alíquota proveniente da mistura das diferentes partes da polpa homogeneizadas em liquidificador, expresso em porcentagem (\%); acidez titulável (AT), determinado conforme o Instituto Adolfo Lutz (1985). Foi retirada uma alíquota de 10 $\mathrm{g}$ de suco, adicionados $40 \mathrm{~mL}$ de água destilada e tituladas com solução de $\mathrm{NaOH}$ a $0,02 \mathrm{~N}$, expresso em porcentagem de ácido málico; relação SS/AT, determinada pela razão entre as duas variáveis SS e AT; açúcares solúveis (AS), determinado conforme Yemn \& Willis (1954), diluindo-se $1 \mathrm{~g}$ de polpa em água destilada até $100 \mathrm{~mL}$, e retirando-se uma alíquota de $100 \mu \mathrm{L}$, adicionados $2 \mathrm{~mL}$ de antrona para as leituras em espectrofotômetro com comprimento de onda de $620 \mathrm{~nm}$, e expressa em percentagem (\%); licopeno, determinado conforme (Rodriguez-Amaya, 2001): utilizou-se 2,5 gramas da amostra adicionados 20 $\mathrm{mL}$ de acetona por um período de uma hora. Foi feita filtragem em funil de separação, onde foram adicionados mais $20 \mathrm{~mL}$ de acetona e $40 \mathrm{~mL}$ de éter de petróleo para extração dos pigmentos. $\mathrm{O}$ extrato foi lavado seis vezes com água destilada. E na solução restante foi adicionado éter de petróleo até $50 \mathrm{~mL}$. O comprimento de onda foi $470 \mathrm{~nm}$ e os resultados expressos em $\mu \mathrm{g} / \mathrm{g}$.

Os dados foram submetidos à análise de variância, teste de Tukey, análise de regressão e análise do desdobramento a $5 \%$ de probabilidade. As análises foram realizadas utilizando o software SISVAR (Ferreira, 2011).

\section{RESULTADOS E DISCUSSÃO}

Para as características físicas (massa média dos frutos, comprimento, diâmetro, espessura de polpa, formato do fruto e firmeza de polpa) não se evidenciou efeito de dose de P, período de aplicação e tempo de armazenamento. Não obstante, houve efeito interativo entre os fatores estudados para as variáveis físico-químicas: açúcares solúveis (AS) e relação SS/AT. Efeito isolado da adubação fosfatada para acidez titulável (AT), AS, relação SS/AT e SS dos frutos e efeito de tempo de armazenamento para perda de massa (PM), SS, AT, AS, relação SS/AT e teor de licopeno.

O peso $(4,09 \mathrm{~kg})$, diâmetro $(19,16$ $\mathrm{cm})$, comprimento $(20,17 \mathrm{~cm})$, espessura de polpa $(17,96 \mathrm{~cm})$ e formato circular dos frutos $(1,08)$ (Tabela 1) não foram afetados pelas doses e formas de aplicação de P. Comportamento semelhante, foi evidenciado em melancia (hibrido Congo) cultivada sob diferentes doses de fósforo $(0,90,180,270$ e $360 \mathrm{~kg} / \mathrm{ha}$ $\mathrm{P}_{2} \mathrm{O}_{5}$ ) por Freitas Junior et al. (2008). Ao contrário, Abrêu et al. (2011) evidenciaram efeito de doses de P no diâmetro dos frutos de melão $(13,15 \mathrm{~cm}$ com 396,8 $\left.\mathrm{kg} / \mathrm{ha} \mathrm{P}_{2} \mathrm{O}_{5}\right)$ e na espessura de polpa $(4,7$ cm com $354,4 \mathrm{~kg} / \mathrm{ha} \mathrm{P}_{2} \mathrm{O}_{5}$ ) e que a massa de frutos aumentou com as doses de $\mathrm{P}$ aplicadas até a dose de $278 \mathrm{~kg} / \mathrm{ha} \mathrm{P}_{2} \mathrm{O}_{5}$.

Sabe-se que a deficiência de $\mathrm{P}$ afeta o desenvolvimento da planta (Mendes et al., 2010), e neste estudo esperava-se que a dose baixa (representada por $50 \%$ da adubação fosfatada utilizada pelos produtores na região) bem como a forma de aplicação de $P$, influenciasse no tama- nho e/ou massa dos frutos. Entretanto, não foi evidenciado efeito deletério desses, o que direciona o produtor para a conveniência e economia no cultivo de melancia, haja vista que a classificação comercial dos frutos, realizada pela massa, não foi prejudicada.

No tempo zero, o teor de AS dos frutos cultivados com dose de $80 \mathrm{~kg} / \mathrm{ha}$ $\mathrm{P}_{2} \mathrm{O}_{5}$ em fundação e com $289 \mathrm{~kg} / \mathrm{ha} \mathrm{P}_{2} \mathrm{O}_{5}$ parcelado utilizando apenas MAP, foram superiores (em torno de 7\%) aos demais tratamentos (Figura 1A). Contudo, ao longo do armazenamento, somente os frutos cultivados com dose de $80 \mathrm{~kg} /$ ha $\mathrm{P}_{2} \mathrm{O}_{5}$ aplicada em fundação apresentaram menor oscilação nos valores de AS, sendo mantidos aos 35 dias de armazenamento, valores próximos aos evidenciado no tempo zero. Por outro lado, os frutos do tratamento com 289 $\mathrm{kg} / \mathrm{ha} \mathrm{P}_{2} \mathrm{O}_{5}$ parcelado utilizando apenas MAP, tiveram diminuição no teor de AS de $4,7 \%$ em relação ao tempo zero. Por outro lado, nos frutos cultivados com 80 e $289 \mathrm{~kg} / \mathrm{ha} \mathrm{P}_{2} \mathrm{O}_{5}$ aplicadas em fundação e cobertura, evidenciaram-se acréscimos de 6,1 e 2,8\% no teor de AS aos 35 dias.

Os açúcares solúveis detectados, neste experimento, tanto ao zero quanto aos 35 dias foram semelhantes aos detectados em melancia 'Crimson Sweet' por Barros et al. (2012) estudando o

Tabela 1. Valores médios de massa (kg), comprimento (Com), diâmetro (DM), espessura de polpa (EP) e formato (F) de frutos da melancia cv. Style em função de diferentes doses, formas de aplicação e fontes de fósforo \{average mass values $(\mathrm{kg})$, length (Com), diameter (DM), pulp thickness (EP) and shape (F) of watermelon fruits, cv. Style depending on different doses, application forms and sources of phosphorus\}. Mossoró, UFERSA, 2014.

\begin{tabular}{lccccc}
\hline Tratamento & Massa (kg) & Com $(\mathbf{c m})$ & DM $(\mathbf{c m})$ & $\mathbf{E P}(\mathbf{c m})$ & $\mathbf{F}$ \\
\hline $\mathrm{T}_{1}$ & $4,08 \mathrm{~A}$ & $20,36 \mathrm{~A}$ & $17,60 \mathrm{~A}$ & $16,42 \mathrm{~A}$ & $1,26 \mathrm{~A}$ \\
$\mathrm{~T}_{2}$ & $4,18 \mathrm{~A}$ & $20,59 \mathrm{~A}$ & $20,00 \mathrm{~A}$ & $18,86 \mathrm{~A}$ & $1,03 \mathrm{~A}$ \\
$\mathrm{~T}_{3}$ & $4,27 \mathrm{~A}$ & $20,46 \mathrm{~A}$ & $19,81 \mathrm{~A}$ & $18,62 \mathrm{~A}$ & $1,03 \mathrm{~A}$ \\
$\mathrm{~T}_{4}$ & $3,73 \mathrm{~A}$ & $19,50 \mathrm{~A}$ & $19,01 \mathrm{~A}$ & $17,72 \mathrm{~A}$ & $1,03 \mathrm{~A}$ \\
$\mathrm{~T}_{5}$ & $4,19 \mathrm{~A}$ & $19,94 \mathrm{~A}$ & $19,36 \mathrm{~A}$ & $18,16 \mathrm{~A}$ & $1,03 \mathrm{~A}$ \\
\hline DMS & 1,2733 & 2,4386 & 4,9031 & 4,8616 & 0,5082 \\
\hline
\end{tabular}

*Médias seguidas de mesma letra na coluna não diferem significativamente entre si pelo teste de Tukey a $5 \%$ de probabilidade (means followed by the same letter in the column do not differ significantly by Tukey test, $5 \%$ ); $\mathrm{T}_{1}=80$ e $\mathrm{T}_{2}=289 \mathrm{~kg} / \mathrm{ha} \mathrm{P}_{2} \mathrm{O}_{5}$ em fundação utilizando superfosfato triplo; $\mathrm{T}_{3}=80 \mathrm{e} \mathrm{T}_{4}=289 \mathrm{~kg} / \mathrm{ha}_{2} \mathrm{O}_{5}$ utilizando superfosfato triplo em fundação e MAP em cobertura; $\mathrm{T}_{5}=289 \mathrm{~kg} / \mathrm{ha}_{2} \mathrm{O}_{5}$ utilizando MAP em fundação e cobertura ( $\mathrm{T} 1=$ 80 and $\mathrm{T} 2=289 \mathrm{~kg} / \mathrm{ha} \mathrm{P}_{2} \mathrm{O}_{5}$ in foundation using triple superphosphate; $\mathrm{T} 3=80$ and $\mathrm{T} 4=289$ $\mathrm{kg} / \mathrm{ha} \mathrm{P}_{2} \mathrm{O}_{5}$ using triple superphosphate in foundation and MAP in coverage; T5= $289(\mathrm{~kg} /$ ha $\mathrm{P}_{2} \mathrm{O}_{5}$ using MAP in foundation and coverage). 
Tabela 2. Valores médios de sólidos solúveis (SS), perda de massa (PM), acidez titulável (AT), firmeza de polpa (FP) e licopeno (L) de frutos da melancia cv. Style em função de diferentes doses, formas de aplicação e fontes de fósforo \{mean values of soluble solids (SS), weight loss (PM), titratable acidity (AT), firmness (FP) and lycopene (L) of watermelon fruit, cv. Style, for different doses, application forms and sources of phosphorus\}. Mossoró, UFERSA, 2014.

\begin{tabular}{llcccc}
\hline Tratamento & SS (\%) & PM (\%) & FP $(\mathbf{N})$ & $\begin{array}{c}\text { AT } \\
(\% \text { Ác. málico) }\end{array}$ & $\begin{array}{c}\mathbf{L} \\
(\boldsymbol{\mu g} / \mathbf{g})\end{array}$ \\
\hline $\mathrm{T}_{1}$ & $9,58 \mathrm{~A}$ & $2,96 \mathrm{~A}$ & $6,35 \mathrm{~A}$ & $0,0956 \mathrm{C}$ & $37,65 \mathrm{~A}$ \\
$\mathrm{~T}_{2}$ & $9,13 \mathrm{~B}$ & $2,89 \mathrm{~A}$ & $6,51 \mathrm{~A}$ & $0,1024 \mathrm{BC}$ & $35,71 \mathrm{~A}$ \\
$\mathrm{~T}_{3}$ & $9,31 \mathrm{AB}$ & $3,32 \mathrm{~A}$ & $7,20 \mathrm{~A}$ & $0,1096 \mathrm{AB}$ & $36,46 \mathrm{~A}$ \\
$\mathrm{~T}_{4}$ & $9,29 \mathrm{AB}$ & $3,14 \mathrm{~A}$ & $6,53 \mathrm{~A}$ & $0,1088 \mathrm{AB}$ & $37,03 \mathrm{~A}$ \\
$\mathrm{~T}_{5}$ & $9,20 \mathrm{AB}$ & $3,36 \mathrm{~A}$ & $6,31 \mathrm{~A}$ & $0,1109 \mathrm{~A}$ & $36,77 \mathrm{~A}$ \\
\hline DMS & 0,4252 & 0,6149 & 1,0020 & 0,0083 & 4,5411 \\
\hline
\end{tabular}

*Médias seguidas de mesma letra na coluna não diferem significativamente entre si pelo teste de Tukey a $5 \%$ de probabilidade (means followed by the same letter in the column do not differ significantly by Tukey test, $5 \%$ ); $\mathrm{T}_{1}=80$ e T $=289 \mathrm{~kg} / \mathrm{ha}_{2} \mathrm{O}_{5}$ em fundação utilizando superfosfato triplo; $\mathrm{T}_{3}=80$ e $\mathrm{T}_{4}=289 \mathrm{~kg} / \mathrm{ha}_{2} \mathrm{O}_{5}$ utilizando superfosfato triplo em fundação e MAP em cobertura; $\mathrm{T}_{5}=289 \mathrm{~kg} / \mathrm{ha}_{2} \mathrm{O}_{5}$ utilizando MAP em fundação e cobertura (T1= 80 and $\mathrm{T} 2=289 \mathrm{~kg} / \mathrm{ha} \mathrm{P}_{2} \mathrm{O}_{5}$ in foundation using triple superphosphate; $\mathrm{T} 3=80$ and T4=289 $\mathrm{kg} / \mathrm{ha} \mathrm{P}_{2} \mathrm{O}_{5}$ using triple superphosphate in foundation and MAP in coverage; T5= $289 \mathrm{~kg} /$ ha $\mathrm{P}_{2} \mathrm{O}_{5}$ using MAP in foundation and coverage).

efeito da aplicação de diferentes doses de nitrogênio na produção e qualidade dos frutos. Também, os resultados obtidos foram semelhantes aos detectados em melancia 'Crimson Sweet' armazenada a 22 e $26^{\circ} \mathrm{C}$ por 12 dias (Araújo Neto et al., 2000).

Geralmente, ocorrem decréscimos nos teores de açúcares, durante o armazenamento, principalmente de frutos que não acumulam reservas de amido, haja vista que são utilizados como substrato respiratório para manutenção do metabolismo celular (Kader, 2002; Taiz \& Zeiger, 2004). Não obstante, em melancia, há evidencias de acúmulo de amido no final do desenvolvimento do fruto (Kim et al., 1998) o que pode explicar o comportamento de acréscimo (nos frutos cultivados com 80 e $289 \mathrm{~kg} /$ ha $\mathrm{P}_{2} \mathrm{O}_{5}$ aplicadas em fundação e cobertura) ou manutenção dos teores de AS nos frutos aos 35 dias de armazenamento.

As doses de $80 \mathrm{~kg} / \mathrm{ha} \mathrm{P}_{2} \mathrm{O}_{5}$ aplicadas em fundação e de 80 e $289 \mathrm{~kg} / \mathrm{ha} \mathrm{P}_{2} \mathrm{O}_{5}$ parceladas, com superfosfato triplo (em fundação) e MAP (em fertirrigação), resultaram em maior teor de AS aos 35 dias de armazenamento $(6,9 \%, 7,0 \%$ e $6,6 \%)$ respectivamente. Porém, a dose de $80 \mathrm{~kg} / \mathrm{ha} \mathrm{P}_{2} \mathrm{O}_{5}$ em fundação é a opção rentável e eficiente para a manutenção dos teores de AS da melancia ao longo do armazenamento.

Para a relação SS/AT dos frutos verificou-se, em todos os períodos de armazenamento, que a dose de $80 \mathrm{~kg} /$ ha $\mathrm{P}_{2} \mathrm{O}_{5}$ aplicada em fundação propiciou maior relação SS/AT dos frutos, com um acréscimo expressivo de $48,33 \%$ quando se compara o tempo zero e 35 dias (Figura 1B). Tal comportamento está diretamente associado a um decréscimo da acidez titulável evidenciado ao longo do período de armazenamento (Figura 2B). Esses valores foram superiores aos detectados por Cecílio Filho \& Grangeiro (2004) em melancia triploide 'Shadow' (46,3 a 51,3), com média de sólidos solúveis de $12,3^{\circ}$ Brix e acidez de $0,25 \%$.

Apesar da relação SS/AT ser indicada para avaliar índice de maturação, essa pode não ser uma medida mais adequada, haja vista que depende do teor de sólidos solúveis e acidez titulável, a não ser que seja estabelecida uma proporção, como foi feita para o melão, em que o melhor sabor foi detectado com proporção igual ou superior a $25: 1$, sendo a acidez máxima de $0,5 \%$ (Cruess, 1973).

Quando comparado, a relação $\mathrm{SS} /$ AT, do tempo zero e 35 dias de armazenamento, verifica-se que todos os tratamentos $\left(80\right.$ e $289 \mathrm{~kg} / \mathrm{ha} \mathrm{P}_{2} \mathrm{O}_{5}$ em fundação, e parcelado em fundação e cobertura, e $289 \mathrm{~kg} / \mathrm{ha} \mathrm{P}_{2} \mathrm{O}_{5}$ parcelado utilizando apenas MAP) apresentaram acréscimos na relação de $48,33 \%$, $20,45 \%, 19,40 \%, 14,06 \%$ e $12,57 \%$, respectivamente. Efeitos de aplicação de fontes de fósforo, também, resultaram em diferenças na relação SS/AT de melão híbrido 'AF-682' (Brito et al., 2000). Porém, neste experimento pode-se constatar que nenhum tratamento resultou em efeito deletério nessa característica, haja vista que a relação SS/ AT foi mantida elevada, mesmo aos 35 dias de armazenamento, com SS acima de $9 \%$ (Figura 2A).

Os tratamentos, doses de $\mathrm{P}$ e tempo de armazenamento, propiciaram efeito isolado no teor de sólidos solúveis dos frutos (Tabela 2). A menor dose de $\mathrm{P}$ $\left(80 \mathrm{~kg} / \mathrm{ha} \mathrm{P}_{2} \mathrm{O}_{5}\right)$ aplicada em fundação propiciou $4,7 \%$ de acréscimo no teor de SS dos frutos em relação a maior dose de $\mathrm{P}\left(289 \mathrm{~kg} / \mathrm{ha} \mathrm{P}_{2} \mathrm{O}_{5}\right)$ aplicada da mesma maneira. Ambos os tratamentos não diferiram significativamente dos demais tratamentos, que por sua vez apresentaram frutos com teor de SS semelhantes entre si. Sendo o teor de SS importante para determinar a qualidade de melancia, evidencia-se efeito positivo no uso de menor dose de P aplicada em fundação, o que na prática representa economia para o produtor, e redução no uso de uma matéria prima escassa (Edixhoven et al., 2013).

Em trabalhos com melão, a adubação fosfatada também influenciou o teor de SS dos frutos (Brito et al., 2000), com melhor efeito para a aplicação de ácido fosfórico até 42 dias após a germinação que o superfosfato simples, aplicado convencionalmente e MAP, aplicado até 30 dias após a germinação.

De acordo com Frydenvang et al. (2015), a deficiência de P, no cultivo de tomateiro, propicia decréscimos leves na concentração de clorofila das folhas, redução na produção de biomassa e presença de antocianina nas margens foliares, bem como maior sistema radicular, o que compromete o ciclo vital da planta. Neste experimento, a menor dose de $\mathrm{P}\left(80 \mathrm{~kg} / \mathrm{ha} \mathrm{P}_{2} \mathrm{O}_{5}\right)$ aplicada em fundação, não causou prejuízo no acúmulo de SS dos frutos, mas viabilizou o uso eficiente do P. 


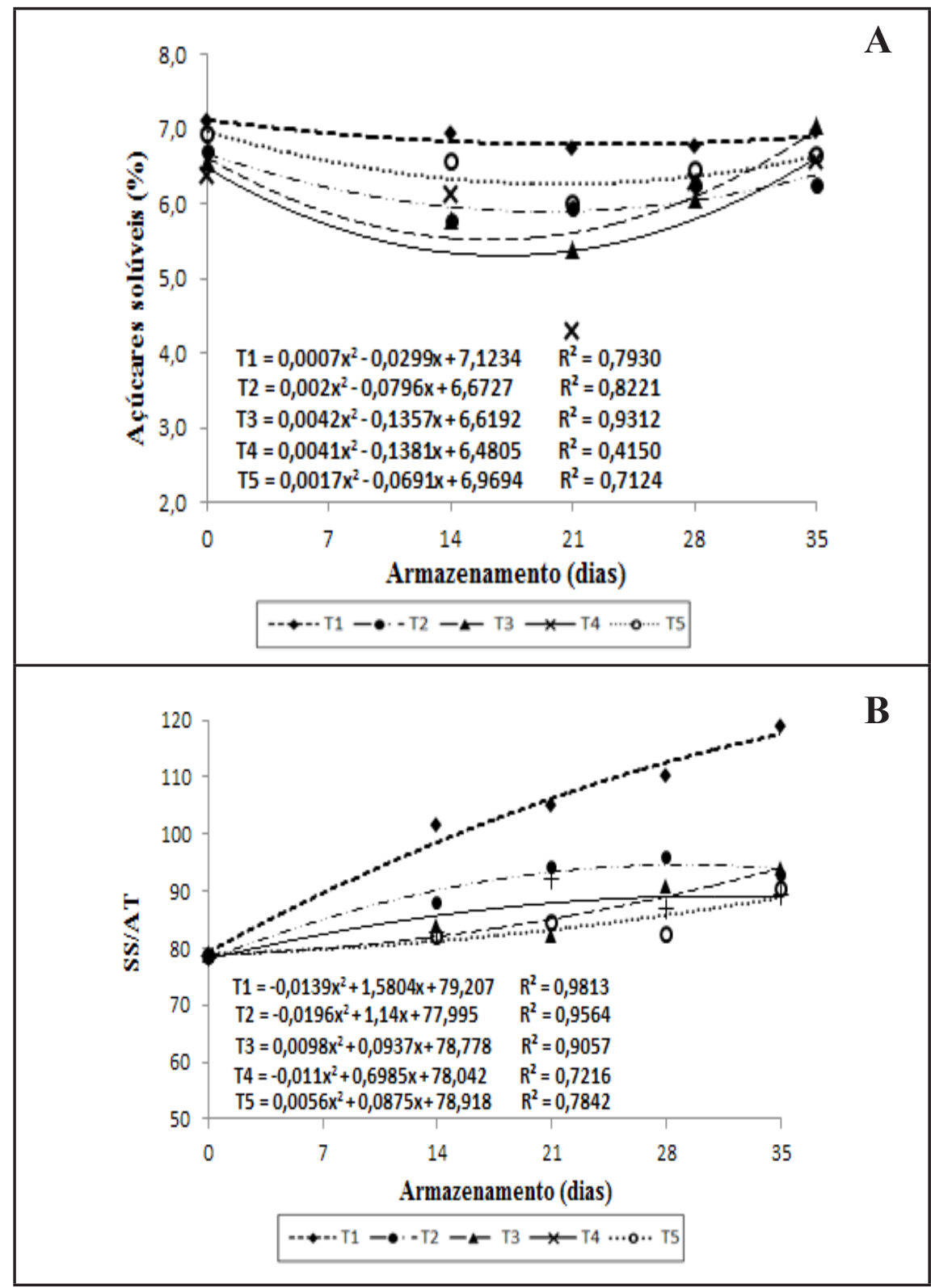

Figura 01. Relação SS/AT (A) e açúcares solúveis (\%) (B) de frutos de melancia 'Style' submetidos a diferentes doses, fontes e formas de aplicação de fósforo em função do período de armazenamento \{SS/AT relation (A) and soluble sugars (\%) (B) of watermelon fruits, cv. Style submitted to different phosphor doses, sources and forms of application, depending on storage period $\} ; \mathrm{T}_{1}=80 \mathrm{e} \mathrm{T}=289 \mathrm{~kg} / \mathrm{haP}_{2} \mathrm{O}_{5}$ em fundação utilizando superfosfato triplo; $\mathrm{T}_{3}=$ 80 e $\mathrm{T}_{4}=289 \mathrm{~kg} / \mathrm{ha} \mathrm{P}_{2} \mathrm{O}_{5}$ utilizando superfosfato triplo em fundação e MAP em cobertura; $\mathrm{T}_{5}=289 \mathrm{~kg} / \mathrm{ha}_{2} \mathrm{O}_{5}$ utilizando MAP em fundação e cobertura $(\mathrm{T} 1=80$ and $\mathrm{T} 2=289 \mathrm{~kg} / \mathrm{ha}$ $\mathrm{P}_{2} \mathrm{O}_{5}$ in foundation using triple superphosphate; T3= 80 and T4 $=289 \mathrm{~kg} / \mathrm{ha} \mathrm{P}_{2} \mathrm{O}_{5}$ using triple superphosphate in foundation and MAP in coverage; T5 $=289\left(\mathrm{~kg} / \mathrm{ha} \mathrm{P}_{2} \mathrm{O}_{5}\right.$ using MAP in foundation and coverage). Mossoró, UFERSA, 2014.

Durante o período de armazenamento, houve acréscimos nos teores de sólidos solúveis dos frutos até os 14 dias $(9,55 \%)$ (Figura $2 \mathrm{~A})$. Porém, houve decréscimos nos valores até 35 dias, entretanto ainda ficaram dentro do limite considerado de boa qualidade comercial $(9,0 \%)$. Tais valores foram superiores aos encontrados por Almeida et al.
(2010), em melancia cultivar 'Quetzali' (8,9\%) e Araújo Neto et al. (2000), em melancia 'Crimson Sweet', cujo teor de SS variou de $7,63 \%$ a $9,55 \%$.

O teor de SS é bastante utilizado para avaliar a qualidade da melancia, é uma medida indireta do teor de açúcares nos frutos. Neste experimento verificou-se que os AS representaram $68,17 \%$ dos
SS. Nota-se que os teores de SS dos frutos apresentaram consonância com o teor de AS, ambos apresentaram decréscimo ao longo do armazenamento, mas aos 35 dias esses se mantiveram próximos aos detectados no tempo zero.

Para a acidez titulável houve efeito isolado de tratamento (Tabela 2) e tempo de armazenamento (Figura 2B). O cultivo com a dose de $289 \mathrm{~kg} / \mathrm{ha} \mathrm{P}_{2} \mathrm{O}_{5}$, parcelado, utilizando apenas MAP em fundação e cobertura, propiciou acidez titulável dos frutos semelhante aos oriundos dos tratamentos 80 e $289 \mathrm{~kg} /$ ha $\mathrm{P}_{2} \mathrm{O}_{5}$ parcelados em fundação e cobertura, mas diferiu daqueles provenientes das doses de 80 e $289 \mathrm{~kg} / \mathrm{ha} \mathrm{P}_{2} \mathrm{O}_{5}$, aplicados em fundação (Tabela 2), sendo os valores superiores a estes, que por sua vez não diferiram entre si. Tal resultado, não implica em grande modificação na qualidade da melancia, em virtude da mesma apresentar acidez muito baixa.

Os ácidos orgânicos dos frutos são sintetizados principalmente através de oxidações, descarboxilações e em alguns casos carboxilações na cadeia respiratória do ciclo de Krebs, e podem ainda ser formados dos açúcares nas fases iniciais da fotossíntese (Kays, 1991).

Abrêu et al. (2011) sugerem que cultivo com doses muito elevadas de $\mathrm{P}$, podem refletir em frutos menos ácidos. As doses utilizadas, neste experimento não foram suficientes para evidenciarmos tal comportamento, entretanto, foi evidenciado efeito de formas de aplicação. Ao contrário, em melão Cantaloupe 'Olimpic express' (Cortez et al., 2011) e Amarelo (Abrêu et al., 2011), cultivados em diferentes doses de $P$, não foi detectado efeito significativo deste na acidez dos frutos.

Ao longo do armazenamento, houve decréscimos de $17,84 \%$ na acidez titulável dos frutos (Figura 2B), mantendo-se aos 35 dias acidez de $0,0967 \%$, fato compreendido haja vista que ácidos orgânicos podem ser utilizados como substratos no processo respiratório (Chitarra \& Chitarra, 2005). Resultados semelhantes foram encontrados por Carlos et al. (2002), em melancia 'Crimson Sweet'; esses observaram um decréscimo de $40,49 \%$ na acidez titulável dos frutos após 30 dias de armazenamento em temperatura de $10^{\circ} \mathrm{C}$. Já Araújo Neto 


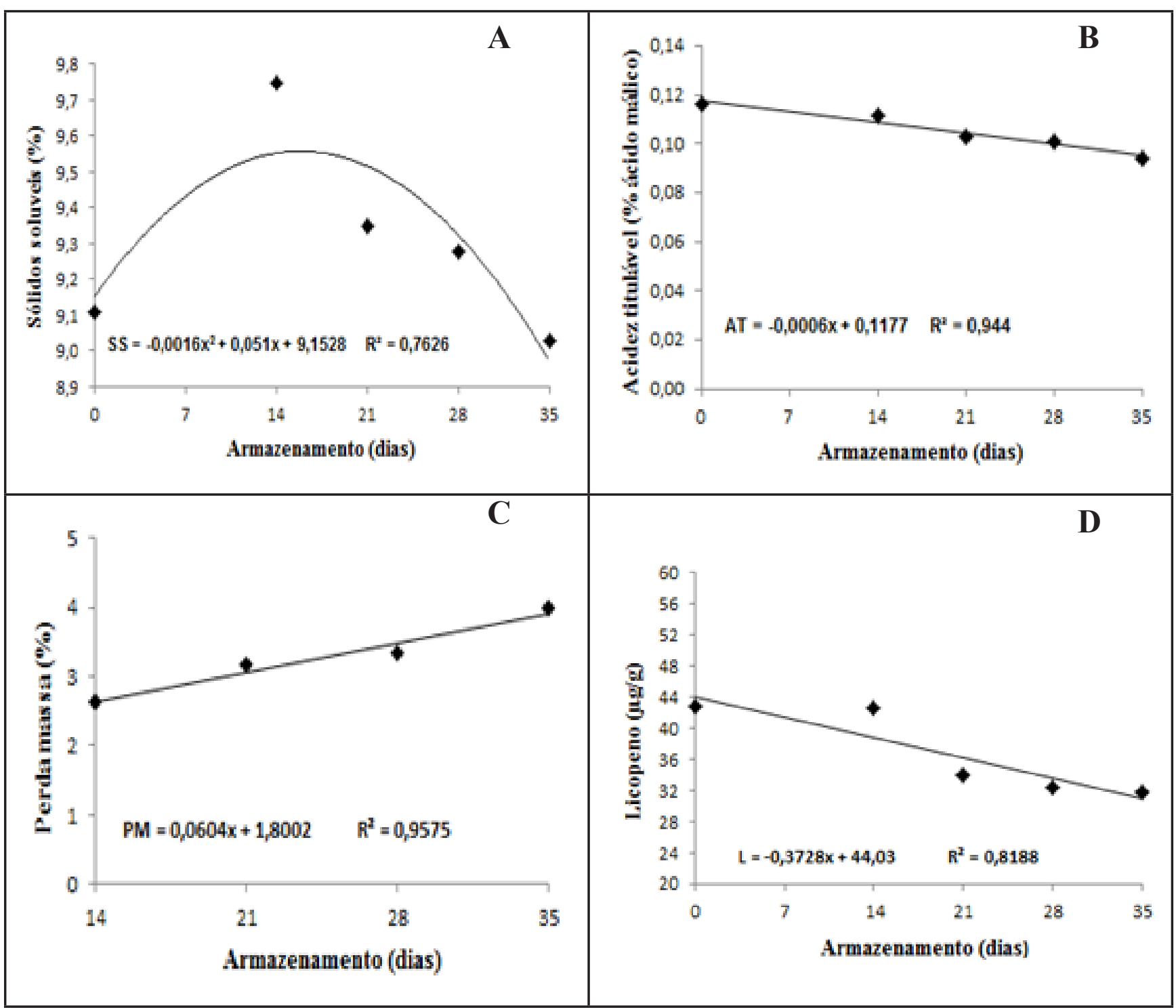

Figura 02. Sólidos solúveis (\%) (A); acidez titulavel (\% de ác. Málico) (B); perda de massa (\%) (C); licopeno ( $\mu \mathrm{g} / \mathrm{g})(\mathrm{D})$ de frutos de melancia 'Style' em função do período de armazenamento \{soluble solids (\%) (A); acidity (\% malic acid) (B); weight loss (\%) (C); lycopene $(\mu \mathrm{g} / \mathrm{g})(\mathrm{D})$ of watermelon fruits, cv. Style, depending on storage period $\}$. Mossoró, UFERSA, 2014.

et al. (2000) e Grangeiro \& Cecílio Filho (2003), detectaram decréscimos na acidez titulável de melancia 'Crimson Sweet' de $58,6 \%$ e $44 \%$ aos 21 e 20 dias de armazenamento em temperatura ambiente, respectivamente.

Os tratamentos não influenciaram a perda de massa dos frutos (Tabela 2), todavia houve acréscimos significativos deste com o tempo de armazenamento (Figura 2C), sendo o maior valor $(3,91 \%)$ observado aos 35 dias. Tal comportamento era de certa forma esperado, já que os frutos não foram embalados.

A perda de massa é uma das principais causas da deterioração e perdas pós-colheita de frutos, por alterar negativamente a aparência e textura (amaciamento, perda de frescor e suculência), tornando-os impróprios à comercialização e consumo (Kader, 2002), e por diminuir o peso dos produtos, resulta em prejuízos econômicos quando comercializados. A perda de massa é resultante, principalmente, do processo de transpiração (Kays, 1991; Kader, 2002; Chitarra \& Chitarra, 2005). Resultados semelhantes foram encontrados em melancia híbrido 'Tide', armazenada (24,7 a $30,4^{\circ} \mathrm{C}$ e 69 a $97 \%$ de UR), durante 20 dias (3,23\%) (Grangeiro \& Cecílio Filho, 2003) e, em melancia 'Crimson Sweet' $(3,79 \%)$ armazenada $\left(24 \pm 2^{\circ} \mathrm{C}\right.$ e $56 \pm 4 \%$ UR) por 21 dias (Araújo Neto et al., 2000).

As melancias apresentaram valores médios de firmeza de polpa de $6,58 \mathrm{Ne}$, não foi evidenciado efeito de tratamento ou tempo de armazenamento nessa característica (Tabela 2). Esse valor foi relativamente baixo se comparado aos valores encontrados por Martins et al. (2013) em melancia 'Quetzali' e 'Style' (13,44 N e 10,63 N, respectivamente) e Carlos et al. (2002) em melancia 'Crimson Sweet' durante 30 dias de armazenamento, com médias de firmeza de 10,92, 12,55 e $12,38 \mathrm{~N}$ em temperatura ambiente, $10 \mathrm{e} 12^{\circ} \mathrm{C}$, respectivamente, tendo os frutos mantido a qualidade comercial por 25 dias em temperatura 
de $10^{\circ} \mathrm{C}$. No entanto, neste experimento constatou-se manutenção da firmeza de polpa das melancias por até 35 dias, atributo importante, pois os tornam mais resistentes às injúrias propicias durante o transporte e a comercialização (Cardoso Neto et al., 2006).

O valor médio de licopeno da polpa de melancia foi de $36,72 \mu \mathrm{g} / \mathrm{g}$ e não variou com o tratamento das doses de P (Tabela 2), mas foi influenciada pelo tempo de armazenamento (Figura 2D). Silva et al. (2011) também não evidenciaram efeito de doses de $\mathrm{P}$ e $\mathrm{K}$ no teor de licopeno de diferentes genótipos de tomate industrial. Em melancia e tomate há acréscimos no teor de licopeno com o amadurecimento, tendo a melancia o teor superior ao tomate e, a melancia sem semente teor superior à melancia com semente (Leskovar et al., 2004).

Houve decréscimos nos teores de licopeno do tempo zero $(44,03 \mu \mathrm{g} / \mathrm{g})$ até 35 dias $(30,98 \mu \mathrm{g} / \mathrm{g})$ de $29,64 \%$. Trata-se de um potente antioxidante, que age bloqueando os radicais livres que danificam as membranas lipoprotéicas (Leskovar et al., 2004), cuja biossíntese em melancia é afetada pela temperatura de armazenamento do fruto argumentam Perkins-Veazie \& Collins (2006), que evidenciaram aumento no teor de licopeno (10-11\%) e $\beta$-caroteno (50-139\%) em polpa de melancia armazenada a $21^{\circ} \mathrm{C}$, enquanto a 5 e $13^{\circ} \mathrm{C}$ não houve mudança significativa nesses carotenóides. Perkins-Veazie \& Collins (2006) evidenciaram também que o aumento do licopeno, $\beta$-caroteno e fitoflueno em melancia mantida a $21^{\circ} \mathrm{C}$ reflete o aumento da atividade de enzimas, envolvida na via da síntese de carotenóides, que são totalmente inibidas pela temperatura de armazenamento de $5^{\circ} \mathrm{C}$.

As doses, forma de aplicação e fontes de $\mathrm{P}$ não afetaram a massa média, comprimento, diâmetro, espessura de polpa, formato do fruto, perda de massa, firmeza de polpa e teor de licopeno dos frutos. Porém, houve efeito de adubação com Pe período de armazenamento no teor de açúcar solúvel. No tempo zero, 14, 21 e 28 dias, os maiores teores de açúcares foram mantidos em frutos cultivados a $80 \mathrm{~kg} / \mathrm{ha} \mathrm{P}_{2} \mathrm{O}_{5}$, aplicado em fundação. Em todos os tratamentos houve decréscimo nos açúcares dos frutos até 21 dias. Os frutos cultivados com a dose de $80 \mathrm{~kg} / \mathrm{ha} \mathrm{P}_{2} \mathrm{O}_{5}$, em fundação, apresentaram maior relação SS/ AT durante o armazenamento. Porém, a relação SS/AT dos frutos de todos os tratamentos aumentou até 35 dias. A perda de massa aumentou e os teores de SS, de acidez e de licopeno diminuíram com o armazenamento dos frutos, mas aos 35 dias os SS mantiveram 9\%. A forma de aplicação, em fundação, na dose de 80 $\mathrm{kg} / \mathrm{ha} \mathrm{P}_{2} \mathrm{O}_{5}$ propiciou maior SS e, a dose de $289 \mathrm{~kg} / \mathrm{ha} \mathrm{P}_{2} \mathrm{O}_{5}$, aplicada parcelada em fundação e cobertura, com apenas MAP, propiciou maior acidez dos frutos.

\section{REFERENCIAS}

ABRÊU, FLG; CAZETTA, JO; XAVIER, TF. 2011. Adubação fosfatada no meloeiroamarelo: reflexos na produção e qualidade dos frutos. Revista Brasileira de Fruticultura 33: 1266-1274.

ALMEIDA, MLB; SILVA, GG; ROCHA, RHC; MORAIS, PLD; SARMENTO, JDA. 2010. Caracterização físico-química de melancia 'quetzali' durante o desenvolvimento. Revista Caatinga 23: 28-31.

ARAÚJO NETO, SE; HAFLE, OM; GURGEL, FL; MENEZES, JB; SILVA, GG. 2000. Qualidade e vida útil pós-colheita de melancia Crimson Sweet comercializada em Mossoró. Revista Brasileira de Engenharia Agrícola e Ambiental 4: 235-239.

BARROS, MM; ARAÚJO, WF; NEVES, LTBC; CAMPOS, AJ; TOSIN, JM. 2012. Produção e qualidade da melancia submetida à adubação nitrogenada. Revista Brasileira de Engenharia Agrícola e Ambiental 16: 1078-1084.

BRITO, LTL; SOARES, JM; FARIA, CMB; COSTA, ND. 2000. Fontes de fósforo aplicadas na cultura do melão via água de irrigação. Revista Brasileira de Engenharia Agrícola e Ambiental 4: 19-22.

CARDOSO NETO, F; GUERRA, HOC; CHAVES, LHG. 2006. Natureza e parcelamento de nitrogênio na qualidade dos frutos do meloeiro. Revista Caatinga 19: 153-160.

CARLOS, ALX; MENEZES, JB; ROCHA, RHC; NUNES, GHS; SILVA, GG. 2002. Vida útil pós-colheita de melancia submetida a diferentes temperaturas de armazenamento. Revista Brasileira de Produtos Agroindustriais 4: 29-35.

CECÍLIO FILHO, AB; GRANGEIRO, LC. 2004. Qualidade de frutos de melancia sem sementes em função de fontes e doses de potássio. Ciência Agrotécnica 28: 570-576.

CHITARRA, MIF; CHITARRA, AB. 2005. Póscolheita de frutos e hortaliças: fisiologia e manuseio. Lavra: UFLA. 785p.

CORTEZ, JWM; CECÍLIO FILHO, AB; GRANGEIRO, LC. 2011. Efeito da adubação fosfatada sobre a qualidade de melão. In: CONGRESSO BRASILEIRO DE OLERICULTURA, 51. Resumos... Viçosa: SOB (CD ROM).

CPRM - SERVIÇO GEOLÓGICO DO BRASIL. 2005. Projeto cadastro de fontes de abastecimento por água subterrânea. Diagnóstico do município de Upanema, estado do Rio Grande do Norte. 10p. Disponível em http://www.cprm.gov.br/rehi/atlas/rgnorte/ relatorios/UPAN169.PDF. Acessado em 09 de julho de 2015.

CRUESS, WV. 1973. Produtos industriais de frutas e hortaliças. São Paulo: Edgard Blucher. $2 \mathrm{v}$.

EDIXHOVEN, JD; GUPTA, J; SAVENIJE, HHG. 2013. Recent revisions of phosphate rock reserves and resources: reassuring or misleading? An in-depth literature review of global estimates of phosphate rock reserves and resources. Earth System Dynamics Discussions 4: 1005-1034.

EMBRAPA. 1999. Sistema brasileiro de classificação de solo. Brasília: Embrapa Solos. 412p.

FENNEMA, OR. 2000. Química de los alimentos. Zaragoza: Acribia,. 1258p.

FERREIRA, DF. 2011. Sisvar: a computer statistical analysis system. Ciência e Agrotecnologia 35: 1039-1042.

FREITAS JUNIOR, NA; BISCARO, GA; SILVA, TRB. 2008. Adubação fosfatada em melancia irrigada, no município de Cassilândia (MS). Cascavel 1: 1-6.

FRYDENVANG, J; MAARSCHALKERWEERD, MV; CARSTENSEN, A; MUNDUS, S; SCHMIDT, SB; PEDAS, PR; LAURSEN, KH; SCHJOERRING, JK; HUSTED, S. 2015. Sensitive detection of phosphorus deficiency in plants using chlorophyll $\alpha$ fluorescence. Plant Physiology 169: 353-361.

GRANGEIRO, LC; CECÍLIO FILHO, AB. 2003. Fontes de potássio e tempo de armazenamento sobre a qualidade pós-colheita de frutos de melancia. In: CONGRESSO BRASILEIRO DE OLERICULTURA, 43. Resumos... Recife: SOB (CD-ROM).

IBGE - Instituto Brasileiro de Geografia e Estatística. 2013. Sistema IBGE de recuperação automática-SIDRA. Disponível em http://www.ibge.gov.br/Acessado em 10 de outubro de 2015.

INSTITUTO ADOLFO LUTZ. 1985. Normas analíticas, métodos químicos e fisicos para análise de alimentos. São Paulo: Instituto Adolfo Lutz. 533p.

KADER, AA. 2002. Postharvest Technology of Horticultural Crops. Wickson Postharvest technology center University of California. $535 \mathrm{p}$.

KAYS, SJ. 1991. Postharvest physiology of perishable plant products. New York: Avi Book. 532p.

KIM, I; KAHNG, H; CHUNG, W. 1998. Characterization of cDNA as encoding small and large subunits of ADP-glucose pyrophosphorylases from watermelon (Citrullus lanatus S.). Bioscience Biotechnology bBiochemical 62: 550-555. 
LESKOVAR, DI; BANG, H; CROSBY, KM; MANESS, N; FRANCO, JA; PERKINSVEAZIE, P. 2004. Lycopene, carbohydrates, ascorbic acid and yield components of diploid and triploid watermelon cultivars are affected by deficit irrigation. J. Hortic. Sci. Biotechnol 79: 75-81.

MACDONALD, GK; BENNETT, EM; POTTER, PA; RAMANKUTTY, N. 2011. Agronomic phosphorus imbalances across the world's croplands. Proceedings of the National Academy of Sciences 108: 3086-3091.

MARTINS, JCP; AROUCHA, EMM; MEDEIROS, JFM; NASCIMENTO, IB; PAULA. VFS. 2013. Características póscolheita dos frutos de cultivares de melancia, submetidas à aplicação de bioestimulante. Revista Caatinga 26: 18-24.

MARTUSCELLI, M; DIMATTIA, C; STAGNARI, F; SPECA, S; PISANTE, M; MASTROCOLA, D. 2015. Influence of phosphorus management on melon (Cucumis melo L.) fruit quality. Journal Science of Food and Agriculture 95: 1-8.

MAYNARD, DN; DUNLAP, AM; SIDOTI, BJ. 2002. Sweetness in diploid and triploid watermelon fruit. Cucurbit Genetics Cooperative Report 25: 32-35.

MENDES, AMS; FARIA, CMB; SILVA, DJ. 2010. Sistema de produção de melancia, adubação. Embrapa Semiárido. Disponível em http://sistemasdeproducao.cnptia. embrapa.br/FontesHTML/Melancia/ SistemaProducaoMelancia/adubacao.htm Acessado em 08 de outubro de 2014.

NOVAIS, RF; SMYTH, TJ; NUNES, FN. 2007. Fósforo. In: NOVAIS, RF; ALVAREZ, VH; BARROS, NF; FONTES, RLF; CANTARUTTI, RB; NEVES, JCL (eds). Fertilidade do solo. Viçosa: Sociedade Brasileira de Ciência de Solo. p. 471-550.

PAULA, JAA; MEDEIROS, JF; MIRANDA, NO; OLIVEIRA, FA; LIMA, CJGS. 2011. Metodologia para determinação das necessidades nutricionais de melão e melancia. Revista Brasileira de Engenharia Agrícola e
Ambiental 15: 911-916.

PRIYA，P; SAHI，SV. 2009. Influence of phosphorus nutrition on growth and metabolism of Duo grass (Duo festulolium). Plant Physiology Biochemistry 47: 31-36.

PERKINS-VEAZIE, P; COLLINS, JK. 2006. Carotenoid changes of intact watermelons after storage. Journal of Agricultural and Food Chemistry. 54: 5868-5874.

RODRIGUEZ-AMAYA, DA. 2001. Guide to carotenoids analysis in food. Washington: International Life Sciences Institute Press. 64p.

SILVA, EC; MACIEL, GM; ALVARENGA, PPM; PAULA, ACCFF. 2011. Teores de $\beta$-caroteno e licopeno em função das doses de fósforo e potássio em frutos de diferentes genótipos de tomateiro industrial. Bioscience J 27: 247-252.

TAIZ, L; ZEIGER, E. 2004. Fisiologia vegetal. Porto Alegre: Artmed. 3. ed.

YEMN, EW; WILLIS, AJ. 1954. The estimation of carbohydrate in plant extracts by antrona. The Biochemical Journal 57: 504-541 